\title{
Palmöl in der EFTA-Indonesien Wirtschaftspartnerschaft
}

\begin{abstract}
Details des neuen Präferenzmechanismus, Informationslage zum Zeitpunkt des Referendums und Gewährleistung der Abstimmungsfreiheit
\end{abstract}

Den EFTA-Staaten ist in den Verhandlungen über eine Wirtschaftspartnerschaft mit Indonesien scheinbar Bahnbrechendes gelungen: Zum ersten Mal weltweit macht ein Handelsabkommen präferenzielle Zölle abhängig von der Art und Weise, wie ein Rohstoff produziert worden ist. Der - theoretische - ökonomische Anreiz zur nachhaltigen Produktion überwindet damit das Verbot in den WTO-Abkommen, zwischen nachhaltig und konventionell produzierten Waren zu unterscheiden. Allerdings zeigt ein näherer Blick, dass die gewährten Präferenzen keinen nennenswerten ökonomischen Wert besitzen. Dass diese Tatsache im Abstimmungskampf nicht offengelegt wurde, wirft Fragen auf: Was ist der Wert der Palmöl-Vereinbarung in der EFTA-Indonesien Wirtschaftspartnerschaft? Hat der Bundesrat seine Pflichten verletzt, indem er unvollständig über den Umfang des ökonomischen Anreizes zur nachhaltigen Palmölproduktion informiert hat? Dieser Beitrag verortet Handlungsbedarf in Bezug auf die Überwindung des Informationsvorsprungs der Bundesverwaltung gegenüber der Legislative im Meinungsbildungsprozess über internationale Handelsabkommen.

I. Einleitung

II. Rechtsinnovation zur Förderung nachhaltiger Produktion durch Handel

III. CEPA-Zollpräferenzen für Palmöl im Detail

IV. Einordnung des CEPA-Präferenzmechanismus

V. Informationslage zum Zeitpunkt des Referendums und Gewährleistung der Abstimmungsfreiheit 277

VI. Schlussfolgerungen

Zitiervorschlag:

CHARLOTTE SIEBER-GASSER, Palmöl in der EFTA-Indonesien Wirtschaftspartnerschaft, sui generis 2021, S. 271

Dr. iur. Charlotte Sieber-Gasser ist Lehrbeauftragte im öffentlichen Recht an den Universitäten Luzern und Zürich und habilitiert an der Universität Bern zur Verfassung der politischen Rechte in der globalisierten Schweiz (charlotte.sieber@gmail.com). Ein herzlicher Dank gebührt Ilona Gremminger vom Staatssekretariat für Wirtschaft SECO für die ausführlichen Diskussionen über den CEPA-Präferenzmechanismus, sowie den beiden anonymen Reviewern für die sorgfältige Durchsicht und wertvollen Inputs.

URL: sui-generis.ch/191

DoI: https://doi.org/10.21257/sg.191

Dieses Werk ist lizenziert unter einer Creative Commons Namensnennung - Weitergabe unter gleichen Bedingungen 4.0 International Lizenz. 


\section{Einleitung}

1 Faktenkenntnis ist für das Funktionieren einer Demokratie unabdingbar. Sie bildet die Grundlage für den politischen Meinungsbildungsprozess und wird im Rahmen von Abstimmungsvorlagen unabdingbar. Und genau hier verfügen Behörden oft über einen Informationsvorsprung. Dieser ist grundsätzlich geeignet, Misstrauen in behördliche Informationstätigkeit zu fördern. Deshalb gelten erhöhte Sorgfaltspflichten für behördliche Informationstätigkeit über Abstimmungsvorlagen.

2 Dass die Behörden bisweilen eine anspruchsvolle Gratwanderung zwischen korrekter, umfassender und einfach verständlicher Information vollbringen müssen, leuchtet ein. Unvollständige oder vereinfachende behördliche Information kann denn auch im Rahmen eines engagierten Abstimmungskampfes von Medien, Interessensvertretungen und Wissenschaft ergänzt werden vorausgesetzt, die der behördlichen Information zugrundeliegende Komplexität wird erkannt. Dass es nicht immer gelingt, den behördlichen Informationsvorsprung im Rahmen eines Abstimmungskampfes aufzuholen, hat sich beispielsweise im Juni 2019 gezeigt in der nachträglichen Aufhebung der Volksabstimmung «Für Ehe und Familie-gegen die Heiratsstrafe»: Das Bundesgericht stellte insbesondere Mängel fest in Bezug auf die Verlässlichkeit der von der Bundesverwaltung genannten Zahlen. ${ }^{1}$ Mangels Nachforschungen durch Medien, Interessensvertretungen und Wissenschaft zu den von der Bundesverwaltung zur Verfügung gestellten Zahlen blieb unerkannt, dass diese eine blosse Schätzung darstellten, lose basierend auf einer Erhebung im Jahr 2001. ${ }^{2}$

3 Der vorliegende Artikel befasst sich mit einer ähnlichen Thematik: Wie sich im Nachgang zum Referendum über dieEFTA-Indonesien Wirtschaftspartnerschaft aufgrund der vorliegenden unabhängigen rechtswissenschaftlichen Fallstudie zeigt, mangelte es der Stimmbevölkerung an für den Meinungsbildungsprozess zentralen Informationen über die Abstimmungsvorlage.

4 Der Artikel erläutert zunächst den Inhalt des genannten Präferenzmechanismus für nachhaltig produziertes Palmöl und geht anschliessend der Frage nach, wer zugewährleisten hat, dass alle Fakten bekannt bzw. zugänglich sind in der Vorbereitungsphase einer Volksabstimmung. Die Problematik bleibt relevant insbesondere im Hinblick auf ähnliche Präferenzmechanismen in weiteren Freihandelsabkommen der Schweiz, aber auch ganz allgemein in Bezug auf behördliche Informationstätigkeit,

1 BGE145I207 E. 3.2. Ähnlich auch BGE138I61 E. 5.2 (Eidg. Volksabstimmung über die Unternehmenssteuerreform).

2 Siehe auch GIOVANNI BIAGGINI, Eine Premiere mit begrenzter präjudizieller Tragweite, ZBI120 2019, S. $531 \mathrm{ff}$. welche zuletzt von verschiedener Seite teils heftig kritisiert worden ist. $^{3}$

\section{Rechtsinnovation zur Förderung nachhaltiger Produktion durch Handel}

Die Wirtschaftspartnerschaft zwischen den EFTA-Staa- 5 ten und Indonesien (Comprehensive Economic Partnership Agreement, $\mathrm{CEPA})^{4}$, führt über Kapitel 8 «Trade and Sustainable Development» weltweit erstmalig Präferenzzölle ein, welche auf nachhaltige Produktion beschränkt sind. Der CEPA-Präferenzmechanismus gilt ausschliesslich für Palmöl. Theoretisch könnte derselbe Mechanismus in Zukunft auch auf andere Rohstoffe und Produkte angewendet werden. Damit besteht das Potential, dass dem CEPA-Präferenzmechanismus Pioniercharakter zukommt und dieser weltweit als Vorbild für weitere Handelspartnerschaften dient.

\section{Artikel 8.10 CEPA}

Massgebend für den erwähnten Präferenzmechanismus 6 ist Art. 8.10 CEPA «Sustainable Management of the Vegetable Oils Sector and Associated Trade». In Art. 8.10 Abs.2 lit. e verpflichten sich die Parteien dazu, sicherzustellen, dass nur nach in CEPA festgelegten Nachhaltigkeitszielen (Art. 8.10 Abs. 2 lit. a) produzierte pflanzliche Öle (inkl. Palmöl) sowie deren Derivate gehandelt werden.

Zusammen mit dem Rest des Kapitels 8 ist Art. 8.10 CEPA 7 von der Streitbeilegung ausgenommen. ${ }^{5}$ Das heisst, dass die Verpflichtungen aus Kapitel 8 nicht über den Rechtsweg durchsetzbar sind und deren Missachtung auch nicht sanktioniert werden kann. Allfällige Uneinigkeiten bei der Umsetzung des Kapitels können im gemischten Ausschuss beraten werden. ${ }^{6}$

Bis hierhin unterscheidet sich das Nachhaltigkeitskapitel 8 in CEPA noch nicht grundlegend von den Nachhaltigkeitskapiteln in modernen Handelspartnerschaften der EU oder auch der USA. Im Gegenteil sehen diese grösstenteils griffigere Durchsetzungsmechanismen vor in Bezug auf die in den Abkommen festgelegten Nachhaltigkeitsziele. ${ }^{7}$

3 Siehe z.B. MICHAEL BOLZLI, Rechtsprofessor Markus Schefer kritisiert Karin Keller-Sutter scharf, nau.ch vom 22. November 2020; VANESSA HANN, Beschwerdeserie gegen das Antiterror-Gesetz-was dahinter steckt in 4 Punkten, watson.ch vom 28. Mai 2021.

4 Comprehensive Economic Partnership Agreement between the Republic of Indonesia and the EFTA States, Jakarta, 16. Dezember 2018 (CEPA).

5 Siehe Art. 8.12 Abs. 3 CEPA.

6 Art. 8.12 Abs. 2 CEPA.

7 Streitbeilegung über ein Schiedsgericht in EU-Abkommen, aber ohne Sanktionsmöglichkeit; Streitbeilegung über ein Schiedsgericht mit 
9 Die eigentliche Rechtsinnovation in CEPA folgt in Annex V, der Verpflichtungsliste der Schweiz über den Import von Rohstoffen und Produkten aus Indonesien, 8 in Verbindung mit der Verordnung über die Einfuhr von nachhaltig produziertem Palmöl aus Indonesien zum Präferenz-Zollansatz (Palmöl-Verordnung). ${ }^{9}$ In Annex V beschränkt die Schweiz den präferenziellen Zugang von Palmöl aus Indonesien teilweise auf nachhaltige Produktion und auf Jahresquoten. ${ }^{10}$ In der Palmöl-Verordnung regelt die Schweiz, wann nachweislich nachhaltig produziertes indonesisches Palmöl von der Zollreduktion erfasst ist.11 Über ein Lieferketten-Zertifizierungssystem wird sichergestellt, dass bei der Einfuhr in die Schweiz zwischen Palmöl mit und ohne Nachhaltigkeitsnachweis unterschieden wird. ${ }^{12}$

10 Dieser Regelungsmechanismus macht einerseits die in Art. 8.10 CEPA festgelegten Nachhaltigkeitsziele durchsetzbar, und macht anderseits zum weltweit ersten Mal einen Präferenzzoll abhängig von der Erfüllung bestimmter nicht-produktbezogener Produktionsmethoden. Indonesisches Palmöl kann somit weiterhin auch ohne Nachhaltigkeitsnachweis in die Schweiz importiert werden. ${ }^{13}$ Es profitiert dann einfach nicht von den CEPAPräferenzen.

\section{Verhältnis zum WTO-Recht}

11 Um vom Meistbegünstigtenprinzip im vorliegend anwendbaren Allgemeinen Zoll- und Handelsabkommen der WTO (General Agreement on Tariffs and Trade, GATT'14) ausgenommen zu sein, müssen Freihandelsabkommen die Voraussetzungen in Art. XXIV GATT erfüllen, das heisst im Wesentlichen Zölle und andere Han-

Sanktionsmöglichkeit in Abkommen der USA; siehe z.B. MARCO BRONCKERS / GIOVANNI GRUNI, Retooling the Sustainability Standards in EU Free Trade Agreements, JIEL 2021, Vol. 24, No.1, S. $25 \mathrm{ff}$.

8 Annex V, Referred to in Article 2.2, Schedule on Tariff Commitments on Goods, Commitments of Switzerland on Goods Originating in Indonesia.

9 Vernehmlassung 2020/73, Verordnung über die Einfuhr von nachhaltig produziertem Palmöl aus Indonesien zum Präferenz-Zollansatz (Palmöl-Verordnung), Frist: 1. April 2021.

10 CEPA, Annex V, (c)-(h), sowie S. 47 ff. Der Meistbegünstigtenzoll is der normale Zolltarif, welcher auf alle Einfuhren von WTO-Mitgliedstaaten Anwendung findet, wenn diese keinen Anspruch auf einen reduzierten Zolltarif haben.

11 Art.1 Palmöl-Verordnung.

12 Art.3-5 Palmöl-Verordnung.

13 Diesbezüglich besteht in Verbindung mit Art. 8.10 Abs. 2 lit. e CEPA, welcher den Handel mit Palmöl zwischen den CEPA Vertragsparteien auf nachhaltige Produktion beschränkt, ein Widerspruch: Um sicherzustellen, dass ausschliesslich nachhaltig produziertes Palmöl gehandelt wird, hätte die Schweiz ein Importverbot auf Palmöl ohne Nachhaltigkeitsnachweis aussprechen müssen.

14 Allgemeines Zoll- und Handelsabkommen, abgeschlossen in Genf am 30. Oktober 1947, für die Schweiz in Kraft getreten am 1. August 1966 (GATT, SR 0.632.21) delsrestriktionen für den Hauptteil des gegenseitigen Aussenhandels («substantially all the trade») beseitigen (Art. XXIV Abs. 8 lit.b GATT). Es reicht im Güterhandel grundsätzlich, dass eine Wirtschaftspartnerschaft Zölle beseitigt bzw. reduziert. Davon abgesehen enthalten die WTO-Abkommen keine weiteren einschränkenden Bedingungen für die Ausgestaltung von Wirtschaftspartnerschaften.

Dies ist wesentlich, weil das WTO-Recht grundsätzlich 12 die Einführung unterschiedlicher Zölle allein basierend auf nicht-produktbezogenen Verfahren und Produktionsmethoden (z.B. Unterscheidung zwischen «fair» und konventionell hergestellter Baumwolle) untersagt. Palmöl aus nachhaltiger auf der einen und aus konventioneller Produktion auf der anderen Seite muss unter WTORecht als «gleichwertig» (like products gemäss Art. III GATT) angesehen werden. Demnach stehen die CEPAPräferenzzölle für nachhaltig produziertes Palmöl grundsätzlich im Widerspruch mit WTO-Recht. ${ }^{15}$ Weil die Präferenzzölle aber allein zwischen den Vertragsparteien der EFTA-Indonesien Wirtschaftspartnerschaft gelten, ist die ansonsten WTO-rechtswidrige Unterscheidung zwischen nachhaltigem und anderem Palmöl dennoch mit den WTO-Verpflichtungen vereinbar.

\section{Signalwirkung für den globalen Markt}

Negative Auswirkungen des globalen Handels auf das 13 Klima, die Umwelt und Arbeitnehmende sind gut dokumentiert und fester Bestandteil des politischen Diskurses weltweit. ${ }^{16}$ Insbesondere die Regulierung von Verfahren und Produktionsmethoden steht dabei im Fokus. Während die Durchsetzung von strengeren Auflagen zum Schutz des Klimas, der Artenvielfalt und Umwelt, sowie der Menschenrechte und Arbeitnehmenden unabdingbar erscheinen mag, ist es nicht ausgeschlossen, dass hinter solchen an und für sich legitimen Forderungen auch eine protektionistische Motivation steckt.

Letzteres ist denn auch - unter anderem - Grund dafür, 14 dass das WTO-Recht grundsätzlich die unterschiedliche Behandlung von Rohstoffen und Produkten basierend auf nicht-produktbezogenen Verfahren und Produktions-

15 Siehe auch THOMAS COTTIER, The Role of PPMs in Extractive Industries, Think Piece, ICTSD und World Economic Forum, Genf 2016, S. 5; RONALD B. DAVIES / KRISHNA CHAITANYA VADLAMANNATI, A race to the bottom in labour standards? An empirical investigation, UCD Centre for Economic Research Working Paper Series, No. WP11/23, Dublin 2011.

16 Siehe z.B. DANI RODRIK, The Globalization Paradox, New York 2011; ALI BURAK GÜVEN, Whither the post-Washington Consensus? International financial institutions and development policy before and after the crisis, Review of International Political Economy 2018, Vol. 25, No. 3, S. $392 \mathrm{ff}$. 
methoden untersagt. ${ }^{17}$ Den WTO-Mitgliedstaaten ist es freigestellt, auf ihrem Staatsgebiet strenge Auflagen zu erlassen. Es ist ihnen allerdings untersagt, diese Auflagen so auszugestalten, dass sie sich extra-territorial - also auf das Staatsgebiet anderer WTO-Mitgliedstaaten - auswirken. ${ }^{18}$ Damit wird ungewollt auch die Erlangung von Wettbewerbsvorteilen über eine Unterschreitung der generell geltenden Auflagen zum Schutz des Klimas, der Umwelt und der Arbeitnehmenden in Verfahren und Produktionsmethoden geschützt. produktbezogene Verfahren und Produktionsmethoden erfüllt somit gleich zwei Anliegen der Industrienationen: 1) Die eigenen Produzentinnen und Produzenten werden davor geschützt, dass sie Wettbewerbsnachteile erleiden aufgrund strengerer Auflagen im Vergleich mit dem Ausland, und 2) der eigene Konsum führt nicht zu einer Verschlechterung der Lebensumstände im Ausland. Auf den ersten Blick ist es den EFTA-Staaten gelungen, ebendiese beiden Anliegen in Bezug auf den Handel mit Palmöl durchzusetzen, ohne dabei auf die Möglichkeit zum Erlass von Handelssanktionen zurückgreifen zu müssen. ferenzmechanismus einerseits auf weitere EFTA-Handelspartnerschaften auswirken wird (z.B. mit Malaysia), anderseits ein ähnlicher Präferenzmechanismus auch in anderen Handelspartnerschaften und in Bezug auf andere Rohstoffe und Produkte diskutiert werden wird. Insbesondere in Bezug auf Palmöl dürfte die Einigung der EFTA-Staaten mit Indonesien dazu beigetragen haben, dass sich längerfristig der Nachhaltigkeitsnachweis in der Palmölproduktion als Standard im globalen Markt durchsetzen wird.

\section{CEPA-Zollpräferenzen für Palmöl im Detail}

17 Beim genaueren Hinsehen entpuppen sich die gewährten CEPA-Präferenzen allerdings als geringfügig. Weil Indonesien als Entwicklungsland in die Gruppe der GSP-

17 Siehe z.B. BERNARD HOEKMAN, Proposals for WTO Reform: A Synthesis and Assessment, Policy Research Working Paper 5525, 2011; CHRISTIANE R. CONRAD, Processes and Production Methods (PPMs) in WTO Law, Cambridge 2011; STEVEN BERNSTEIN / ERIN HANNAH, Non-State Global Standard Setting and the WTO: Legitimacy and the Need for Regulatory Space, JIEL 2008, Vol.11, No. 3, S. $590 \mathrm{ff}$.

18 Ausser eine solche Auflage liesse sich über eine der allgemeinen Ausnahmen in den WTO-Abkommen rechtfertigen. Siehe dazu auch CHARLOTTE SIEBER-GASSER / SMRITI KALRAS / ADITI VISHWAS-SHETH, Sustainable Development Goals vs Non-Discrimination in WTO Law: Does the End Justify the Means?, Indian Journal of International Economic Law, Mai/Juni 2021.
Länder ${ }^{19}$ fällt, sind Einfuhren von Palmöl bereits heute teilweise zollbefreit. ${ }^{20}$ Zudem finden abhängig vom jeweiligen Verwendungszweck Zollbegünstigungscodes ${ }^{21}$ Anwendung - auch ohne Abkommen.

\section{CEPA Annex $\mathrm{V}$}

In CEPA Annex V finden sich die genannten Zollreduk- 18 tionen auf nachweislich nachhaltig produziertes Palmöl. Sie sind als fixer Betrag in CHF angegeben, welcher vom jeweils aktuell angewendeten Meistbegünstigtentarif abgezogen wird. ${ }^{22}$ Weil sich der angewendete Meistbegünstigtentarif ändern kann, verändert sich damit auch der Prozentsatz der gewährten Zollreduktion. Aktuell bewegen sich die gewährten Zollreduktionen im Rahmen von $20-40 \%$.

Die gewährten Zollreduktionen sind über Quoten auf ein 19 Jahrestotal an Einfuhren gebunden. ${ }^{23}$ Überschreiten die Einfuhren in einem Jahr die in Annex V festgelegten Quoten, wird die Zollreduktion für den verbleibenden Rest des Jahres aufgehoben.

Schliesslich hält Annex V fest, dass allfällig gewährte zu- 20 sätzliche Präferenzen gegenüber einem anderen Palmölproduzierenden Staat auch auf Indonesien Anwendung finden. ${ }^{24}$ Dies ist aktuell insbesondere mit Blick auf die laufenden Verhandlungen mit Malaysia von Bedeutung: Sollte die Schweiz Malaysia einen besseren Marktzugang für Palmöl gewähren, müsste sie die entsprechenden Präferenzen auch auf Indonesien ausdehnen.

\section{Tatsächliche Zollpräferenzen}

Im Detail ergeben sich folgende Bestimmungen über Zoll- 21 präferenzen gegenüber Einfuhren von Palmöl aus Indonesien (aufgeführt als Meistbegünstigtentarif(MFN Zoll), Begünstigungscode (MFN Code), CEPA Zoll, Quote falls anwendbar und tatsächliche Menge an Import im Durchschnitt 2017-2020):25

19 Generalised System of Preferences (GSP). Siehe Eidg. Zollverwaltung, Entwicklungsländer APS/GSP (Generalised System of Preferences), Länder und Zollpräferenzen. Nach Ratifikation des CEPA ersetzt CEPA die GSP-Präferenzen für Indonesien.

20 Einsehbar unter Eidg. Zollverwaltung, Schweizerischer Gebrauchs tarif, Zollveranlagung: www.tares.ch.

21 Waren, die auf Grund ihrer Verwendung zu einem reduzierten Zollansatz ins Zollgebiet verbracht werden, siehe Art.14 Zollgesetz vom 18. März 2005 (ZG; SR 631.0); Art. 50 ff. Zollverordnung vom 1. November 2006 (ZV; SR 631.01); Verordnung des EFD über Zollerleichterungen für Waren je nach Verwendungszweck vom 4. April 2007 (Zollerleichterungsverordnung; SR 631.012).

22 Litera (b) Annex V CEPA.

23 Literae (c)-(h) Annex V CEPA.

24 Litera (j) Annex V CEPA.

25 Angaben in CHF/100 kg, Stand April 2021. Quellen: Annex V CEPA, Eidg. Zollverwaltung (www.tares.ch). 


\begin{tabular}{|c|c|c|c|c|c|c|}
\hline Zolllinie & Verwendungszweck & MFNZoll & MFN Code & CEPA Zoll & CEPA Quote & Import/Jahr ${ }^{26}$ \\
\hline & crude palm oil: & & & & & 0 \\
\hline 1511.101 & for animal feeding & $\overline{0}$ & - & $\overline{0}$ & & 6.2 \\
\hline \multirow[t]{5}{*}{1511.109} & for technical purposes & 116.05 & $\overline{1}$ & $79.35 / 0$ & $\mathrm{~A} / \mathrm{D}$ & \\
\hline & for the manufacture of soups & 116.05 & 0.1 & - & & \\
\hline & for the manufacture of spreads & 116.05 & $\overline{6}$ & - & & \\
\hline & for the industrial manufacture of condiments & 116.05 & 1 & $\overline{0}$ & & \\
\hline & fusion point higher than that of palm oil: & & & & & \\
\hline$\overline{1511.9011}$ & for animal feeding & 0 & - & $\overline{0}$ & & 157 \\
\hline \multirow[t]{7}{*}{$\overline{1511.9018}$} & refined oil in tanks or metal drums: & & & & & 0 \\
\hline & for further refining & 157.25 & 132.7 & $91.45 / 124.35$ & $\mathrm{~B} 1 / \mathrm{B} 2$ & \\
\hline & for the manufacture of edible fats/oils & 157.25 & 137.65 & $\overline{91.45 / 124.35}$ & $\mathrm{~B} 1 / \mathrm{B} 2$ & \\
\hline & for technical purposes & 157.25 & $\overline{1}$ & 0 & & \\
\hline & for the industrial manufacture of soups & 157.25 & 10 & $\overline{0}$ & & \\
\hline & for further refining of soups & 157.25 & 0.1 & - & & \\
\hline & for the industrial manufacture of condiments & 157.25 & 1 & $\overline{0}$ & & \\
\hline \multirow[t]{5}{*}{1511.9019} & refined oil other: & & & & & 1.6 \\
\hline & for the manufacture of edible fats/oils & 168.1 & 138.3 & $115.3 / 0$ & $\mathrm{~A} / \mathrm{D}$ & \\
\hline & for technical purposes & 168.1 & 1 & 0 & & \\
\hline & for the industrial manufacture of soups & 168.1 & $\overline{10}$ & - & & \\
\hline & for the industrial manufacture of condiments & 168.1 & 1 & 0 & & \\
\hline$\overline{1511.9091}$ & for animal feeding & $\overline{0}$ & - & $\overline{0}$ & & 3 \\
\hline \multirow[t]{6}{*}{1511.9098} & refined oil other in tanks or metal drums: & & & & & 6 \\
\hline & for the manufacture of edible fats/oils & 138.25 & 133.15 & $94.55 / 0$ & $\mathrm{~A} / \mathrm{D}$ & \\
\hline & for technical purposes & 138.25 & $\overline{1}$ & 0 & & \\
\hline & for the industrial manufacture of soups & 138.25 & 10 & 0 & & \\
\hline & for further refining of soups & 138.25 & 0.1 & - & & \\
\hline & for the industrial manufacture of condiments & 138.25 & 1 & 0 & & \\
\hline \multirow[t]{4}{*}{1511.9099} & refined oil other other: & & & & & 0.3 \\
\hline & for technical purposes & 149.1 & 1 & 0 & & \\
\hline & for the industrial manufacture of soups & 149.1 & 10 & $102 / 0$ & $\mathrm{~A} / \mathrm{D}$ & \\
\hline & for the industrial manufacture of condiments & 149.1 & 1 & 0 & & \\
\hline 1513.219 & crude palm kernel oil: & & & & & 0 \\
\hline \multirow[t]{4}{*}{1513.211} & for animal feeding & $\overline{0}$ & - & $\overline{0}$ & & 0 \\
\hline & for technical purposes & 121.6 & 1 & 0 & & \\
\hline & for the manufacture of spreads & 121.6 & 6 & $70.3 / 96$ & $\mathrm{C} 1 / \mathrm{C} 2$ & \\
\hline & for the industrial manufacture of condiments & 121.6 & 1 & 0 & & \\
\hline \multirow[t]{4}{*}{1513.2918} & fusion point higher than that of palm kernel oil in tanks: & & & & & 0 \\
\hline & for the manufacture of edible fats/oils & 157.25 & 147.35 & $91.45 / 124.35$ & $\mathrm{C} 1 / \mathrm{C} 2$ & \\
\hline & for technical purposes & 157.25 & 1 & 0 & & \\
\hline & for the industrial manufacture of condiments & 157.25 & $\overline{1}$ & $\overline{0}$ & & \\
\hline \multirow[t]{4}{*}{1513.2919} & fusion point higher than that of palm kernel oil other: & & & & & 0 \\
\hline & for technical purposes & 168.1 & $\overline{1}$ & 0 & & \\
\hline & for the industrial manufacture of condiments & 168.1 & $\overline{1}$ & $\overline{0}$ & & \\
\hline & unidentified & 168.1 & - & $97.7 / 132.9$ & $\mathrm{C} 1 / \mathrm{C} 2$ & \\
\hline \multirow[t]{4}{*}{1513.2998} & refined palm kernel oil in tanks or metal drums: & & & & & 0 \\
\hline & for the manufacture of edible fats/oils & 145.25 & 140.5 & $84.25 / 114.75$ & $\mathrm{C} 1 / \mathrm{C} 2$ & \\
\hline & for technical purposes & 145.25 & 1 & 0 & & \\
\hline & for the industrial manufacture of condiments & 145.25 & 1 & 0 & & \\
\hline \multirow[t]{4}{*}{1513.2999} & refined palm kernel oil other: & & & & & 0 \\
\hline & for technical purposes & 156.1 & $\overline{1}$ & $\overline{0}$ & & \\
\hline & for the industrial manufacture of condiments & 156.1 & $\overline{1}$ & 0 & & \\
\hline & unidentified & 156.1 & - & $90.5 / 123.3$ & $\mathrm{C} 1 / \mathrm{C} 2$ & \\
\hline
\end{tabular}

26 Angaben in Tonnen/Jahr, im Durchschnitt, 2017-2020. Aussenhandelsstatistik, Swiss-Impex Datenbank der Eidg. Zollverwaltung EZV. 

Nachhaltigkeitspräferenzen im Rahmen von $\mathrm{CHF} 1 / 100 \mathrm{~kg}$ (in hellgrau). Gegeben, dass $100 \mathrm{~kg}$ Palmöl in der Schweiz ca. CHF 100 kosten ${ }^{27}$ und dass zum heutigen Zeitpunkt ungewiss ist, inwiefern der Nachhaltigkeitsnachweis zu zusätzlichen Kosten (inkl. Zeitaufwand) führen wird, erscheint die potentielle Ersparnis von $\mathrm{CHF} 1 / 100 \mathrm{~kg}$ vernachlässigbar. lich Palmöl für die Produktion von Futtermittel in die Schweiz importiert hat und diese Einfuhren generell zollbefreit sind. ${ }^{28}$ Die Schweiz konnte deshalb in Bezug auf Palmöl-Importe für Futtermittel keine zusätzlichen Präferenzen im Gegenzug zum Nachhaltigkeitsnachweis anbieten. Basierend auf der bisherigen, relativ konstanten Zusammensetzung der Palmöl-Importe aus Indonesien ist somit davon auszugehen, dass bis deutlich über 90\% des indonesischen Palmöls vom CEPA-Nachhaltigkeitsnachweis gar nicht erfasst ist (dunkelgrau in der Kolonne «MFN Zoll»). tigkeitspräferenzen auf einzelne Zolllinien (dunkelgrau in der Kolonne «CEPAZoll»), wobei Indonesien über diese Zolllinien in der Vergangenheit kaum oder gar kein Palmöl in die Schweiz importiert hat.

\section{Einordnung des CEPA-Präferenzmechanismus}

26 Gegeben, dass damit die CEPA-Zollreduktionen kaum oder gar nicht Anwendung finden werden, bleibt die Frage, wie der Wert des CEPA-Präferenzmechanismus insgesamt einzuordnen ist. Dieser Wert ergibt sich nicht direkt und allein aus dem Abkommenstext:29

\footnotetext{
27 Aussenhandelsstatistik, Swiss-Impex Datenbank der Eidg. Zollverwaltung EZV.

28 Siehe HandelsstatistikJahre 2017-2020: Zwischen 73.5\% und 99\% der Importe aus Indonesien waren zollbefreit und für die Produktion von Futtermittel bestimmt. Aussenhandelsstatistik, Swiss-Impex Datenbank der Eidg. Zollverwaltung EZV.

29 Ähnlich auch ELISABETH BÜRGI BONANOMI, Die Nachhaltigkeit im Handelsabkommen mit Indonesien mit besonderem Fokus auf die Regulierung des Palmöl-Imports, Rechtsgutachten im Auftrag der Agrarallianz Schweiz, 15. März 2019.
}

1. Indonesien erhält die Zusicherung, dass unabhängig von den Entwicklungen in der GSP-Ländergruppe und in den Zollbegünstigungscodes auf jeden Fall die CEPA-Zollreduktionen Anwendung finden (fallback option).

2. Indonesien erhält zudem die Zusicherung, dass sämtliche zukünftigen weiterreichenden Präferenzen der Schweiz auch auf indonesische Einfuhren ausgedehnt werden (Meistbegünstigtenprinzip).

3. Die Schweiz erhält das Recht, den Nachhaltigkeitsnachweis über Einfuhrkontrollen durchzusetzen.

4. Der CEPA-Präferenzmechanismus erhöht den politischen Druck auf Palmöl-Produzentinnen und -Produzenten, von der konventionellen zur nachhaltigen Produktion zu wechseln.

Zudem ist der CEPA-Präferenzmechanismus geeignet, 27 den politischen Druck auf andere WTO-Mitgliedstaaten zu erhöhen, ähnliche Präferenzmechanismen in ihre Handelspartnerschaften aufzunehmen. Je mehr WTOMitgliedstaaten ähnliche Vereinbarungen treffen, desto eher entsteht ein globaler Konsens über nicht zu unterschreitende Mindestauflagen. ${ }^{30}$ Insbesondere, weil sich die ökonomischen Anreize des CEPA-Präferenzmechanismus bei näherem Hinsehen als geringfügig herausstellen, liegt es durchaus im Rahmen des Möglichen, dass ein solcher «regulatory spill-over effect» eintritt: Während politisch ein Zeichen gesetzt wird, wird dieses ökonomisch nur über einen «nudge» durchgesetzt.

Der CEPA-Präferenzmechanismus liesse sich im Grund- 28 satz auch auf andere Produkte und Rohstoffe übertragen, sofern die folgenden Voraussetzungen erfüllt sind:

1. Es handelt sich um ein klar eingrenzbares, spezifisches Anliegen, welches ein spezifisches Produkt oder einen spezifischen Rohstoff betrifft (hier: keine weitere Abholzung durch bilateralen Handel mit Palmöl).

2. Ein passender Standard, welcher dem Anliegen gerecht wird, ist bereits etabliert (hier: z.B. Roundtable on Sustainable Palm Oil, RSPO) ${ }^{31}$.

3. Strukturen und Verfahren zur Überprüfung der Einhaltung des bestehenden Standards sind bereits etabliert (hier: Verfahren zur RSPO-Zertifizierung).

4. Der bestehende Meistbegünstigtentarif ist nicht 0 .

30 THOMAS COTTIER / CHARLOTTE SIEBER-GASSER/GABRIELA WERMELINGER, The Dialectical Relationship of Preferential and Multilateral Trade Agreements, in: Dür/Elsig (Hrsg.), Trade Cooperation: The Purpose, Design and Effects of Preferential Trade Agreements, Cambridge 2015, S. 465ff.

31 Globaler Standard für nachhaltig produziertes Palmöl, siehe Roundtable on Sustainable Palm Oil, RSPO Certification. 

den Strukturen und Verfahren, welche die Einhaltung des Standards zuverlässig garantieren, funktioniert der CEPA-Präferenzmechanismus nicht. Entsprechend baut der CEPA-Präferenzmechanismus faktisch auf die bestehende Nachfrage nach einem etablierten privaten Standard auf und integriert diesen formell in ein Handelsabkommen. ge» darstellt, muss der Import des betreffenden Produkts oder Rohstoffs in substantiellem Umfang verzollt sein. Ist der Import - wie bei Palmöl für die Produktion von Futtermitteln - bereits zollfrei oder nur in geringfügigem Mass zollpflichtig, fällt die Möglichkeit zur Gewährung einer Zollpräferenz entweder ganz weg oder mindert sich zu einem «nudge». Damit kommen nur die vergleichsweise wenigen verbleibenden Produkte und Rohstoffe für einen griffigen CEPA-Präferenzmechanismus in Frage, welche weiterhin stark zollpflichtig sind. Diese umfassen in der Schweiz primär Landwirtschaftsgüter, denn der Import von Industriegütern ist typischerweise (quasi) zollfrei. ${ }^{32}$

31 Der CEPA-Präferenzmechanismus birgt aber selbstverständlich auch die Gefahr, dass er unter dem Deckmantel der Nachhaltigkeit primär dem Protektionismus dient: Augenscheinlich dient er auch dem Schutz der heimischen Produktion von Pflanzenöl. ${ }^{33}$ Ist aber die Nachfrage unabhängig von staatlicher Intervention bereits klar positioniert - in der Schweiz besteht in der Nahrungsmittelindustrie so gut wie keine Nachfrage nach nicht RSPO-zertifiziertem Palmöl ${ }^{34}$ - entschärft dies bis zu einem gewissen Grad den protektionistischen Ansatz des CEPA-Präferenzmechanismus: Er bindet dann, was bereits marktüblich ist (sozusagen nach dem Vorbild einer «ratchet-clause»).

Damit ist der CEPA-Präferenzmechanismus Beweis dafür, dass das private Konsumverhalten die Struktur internationaler Handelsbeziehungen prägt, und dass private Standards - wenn einmal etabliert - zum neuen allgemein gültigen Standard erhoben werden können. Der CEPA-

32 Siehe KOEN BERDEN / ANIRUDH SHINGAL / CHARLOTTE SIEBER-GASSER, Freihandelsabkommen: Verhandlungsposition nur leicht betroffen, Die Volkswirtschaft 4/2018, S.14ff.

33 Economiesuisse, Warum das Ausklammern von Palmöl in Freihan delsverhandlungen nicht zielführend ist, Faktenblatt: Palmöl; CHRISTIAN FELBER, Freihandelsabkommen mit Malaysia. Konsequenzen für den Schweizer Agrar- und Lebensmittelsektor, Bachelor-Thesis, Olten 2016; Schweizer Bauer vom 25.Januar 2021 (Palmöl: Bauernverband für Freihandelsabkommen).

34 Staatssekretariat für Wirtschaft SECO, Umfassendes Wirtschafts partnerschaftsabkommen (CEPA) EFTA-Indonesien: «Bereits heute ist fast das gesamte für die Schweizer Lebensmittelindustrie bestimmte Palmöl gemäss Nachhaltigkeitsstandards zertifiziert.»
Präferenzmechanismus entspricht somit dem sich im Bereich der globalen Nachhaltigkeitspolitik durchsetzenden «bottom-up» Ansatz, kann aber nicht eigenständig als eigentlicher Treiber des Wandels zu nachhaltigerem Handel bezeichnet werden. ${ }^{35}$

\section{Informationslage zum Zeitpunkt des Referendums und Gewährleis- tung der Abstimmungsfreiheit}

Während der de jure zahnlose CEPA-Präferenzmecha- 33 nismus für die Ausgewogenheit der EFTA-Indonesien Wirtschaftspartnerschaft insgesamt unproblematisch erscheint, wirft die offizielle Darstellung davon grundsätzliche Fragen auf in Bezug auf die allgemeine Informationslage zum Zeitpunkt der Abstimmung. Gegen die EFTAIndonesien Wirtschaftspartnerschaft wurde im Januar 2020 das Referendum ergriffen, welches im Juli 2020 auch zustande kam. ${ }^{36}$ Es ist dies das erste Referendum über ein Freihandelsabkommen seit der Abkehr von der sog. Standardabkommen-Praxis und überhaupt erst die zweite Volksabstimmung über ein Freihandelsabkommen in der Geschichte der Schweiz. ${ }^{37}$ Vier der fünf zentralen vom Referendumskomitee aufgeführten Gründe gegen die EFTA-Indonesien Wirtschaftspartnerschaft betrafen den Handel mit Palmöl. Auf der entsprechenden Webseite wird das Referendum als «Referendum Stop Palmöl» bezeichnet. ${ }^{38}$ In der Folge fokussierte sich der Abstimmungskampf zum Referendum vom 7. März 2021 auf die Bestimmungen rund um die Einfuhr von indonesischem Palmöl in die Schweiz. Dabei wurde der Komplexität des CEPA-Präferenzmechanismus kaum Rechnung getragen.

Möglicherweise war es nicht im Interesse der verschiede- 34 nen Interessengruppen, sich im Abstimmungskampf allzu sehr in die Details des Präferenzmechanismus zu vertiefen: 1) Das Referendumskomitee hätte aufgrund der hier aufgeführten Sachlage eingestehen müssen, dass der Grossteil der Palmöl-Importe aus Indonesien für die Futtermittelproduktion bestimmt ist, was bisher die Konsumentinnen und Konsumenten in der Schweiz nicht gestört hat. Der zollfreie Import von Palmöl zu Futtermittelzwecken liegt zudem im direkten Interesse der schweizerischen Landwirtschaft: Über den zollfreien Import

35 Siehe z.B. FRANK BIERMANN / NORICHIKA KANIE / RAKHYUN E. KIM, Global Governance by Goal-setting: The Novel Approach of the UN Sustainable Development Goals, Current Opinion in Environmental Sustainability 2017, S. $26 \mathrm{ff}$.

36 Bundeskanzlei, Referendum gegen das Wirtschaftspartnerschaftsabkommen mit Indonesien zustande gekommen, 2. Juli 2020.

37 Siehe z.B. LOUIS GEBISTORF, Ist die Standardabkommen-Praxis Geschichte?, sui generis 2020.

38 Referendumskomitee gegen CEPA, Stop Palmöl, 5 Gründe gegen das schädliche Freihandelsabkommen. 
sind Futtermittel günstiger. 2) Auch die Gegenseite hatte kein Interesse daran offenzulegen, dass der Präferenzmechanismus letztlich nur max. 10\% der ohnehin bereits geringfügigen Palmöl-Importe aus Indonesien erfasst, denn damit hätte das Argument, es sei Bahnbrechendes gelungen, an Bedeutung verloren.

Die behördliche Informationstätigkeit allerdings muss den Grundsätzen der Sachlichkeit, Transparenz und Verhältnismässigkeit genügen. Sie erfüllt damit die wichtige Aufgabe, die Faktenlage zu klären, wenn sich politische Akteure ansonsten im Sinne ihrer jeweiligen Zielsetzungen nicht unbedingt nur an Fakten orientieren. Im vorliegenden Beispiel wäre somit der behördlichen Informationstätigkeit die Aufgabe zugekommen, die Fakten zum Präferenzmechanismus neutral und transparent zu klären.

\section{Abstimmungsfreiheit}

Art. 34 Abs. 2 BV ${ }^{39}$ garantiert, dass kein Abstimmungsresultat anerkannt wird, welches nicht den freien Willen der Stimmberechtigten zum Ausdruck bringt. Dies setzt einen umfassenden und unbehinderten Meinungsbildungsprozess voraus. ${ }^{40}$ Abstimmungsinformationen müssen objektiv sein und die wesentlichen Gesichtspunkte enthalten, ${ }^{41}$ und den Grundsätzen der Sachlichkeit, Transparenz und Verhältnismässigkeit genügen. ${ }^{42}$ Verletzt ist die Pflicht zur Sachlichkeit, wenn Behörden über Zweck und Tragweite einer Vorlage falsch orientieren oder sie wesentliche, für die Meinungsbildung bedeutsame Umstände nicht thematisieren. ${ }^{43}$ Die Pflicht zur Transparenz ist verletzt, wenn behördliche Information nicht als solche erkennbar ist. ${ }^{44}$ Das Gebot der Verhältnismässigkeit schliesslich ist verletzt, wenn die behördliche Informationstätigkeit den Abstimmungskampf dominiert. 45

39 Bundesverfassung der Schweizerischen Eidgenossenschaft vom 18. April 1999 (BV; SR101).

40 GIOVANNI BIAGGINI et al., Staatsrecht, 2. Aufl., St. Gallen et al. 2015 S. 601; RENÉ RHINOW / MARKUS SCHEFER / PETER UEBERSAX, Schweizerisches Verfassungsrecht, 3. Aufl., Basel 2016, para. 2063; ULRICH HÄFELIN / WALTER HALLER / HELEN KELLER / DANIELA THURNHERR, Schweizerisches Bundesstaatsrecht, 9. Aufl., Zürich et al. 2016, para.1387; BGE125I441; BGE124 I 55; BGE145I 282 E. 4.1

41 BGE132I104 E. 4; PASCAL MAHON, Les droits politiques, in: Diggel mann/Hertig Randall/Schindler (Hrsg.), Verfassungsrecht der Schweiz, Band II, Zürich 2020, S.1531ff.

42 Art.10 $a$ Bundesgesetz über die politischen Rechte vom 17. Dezember 1976 (BPR; SR 161.1); RHINOw et al. (Fn. 40), para. 2079; BGE140 I 338; PIERRE TSCHANNEN, in: Waldmann/Belser/Epiney (Hrsg.), Bundesverfassung, Basler Kommentar, Basel 2015, Art. 34 BV, para. 33 (zit. BSK BV-TSCHANNEN).

43 RHINOw et al. (Fn. 40), para. 2079b.

44 RHINOw et al. (Fn. 40), para. 2081a; BGE114 Ia 427; BGE132 I 104.

45 RHINOw et al. (Fn. 40), para. 2082.
Private sind im Gegensatz zu den Behörden im Abstim- 37 mungskampf nicht an die Grundsätze der Sachlichkeit, Transparenz und Verhältnismässigkeit gebunden. Bei Abstimmungen obliegt daher den Behörden auch eine Schutzpflicht, offensichtlich falsche Informationen richtigzustellen. ${ }^{46}$ Private Falschinformationen können die Abstimmungsfreiheit verletzen, wenn es «dem Bürger nach den Umständen unmöglich ist, sich aus anderen Quellen ein zuverlässiges Bild von den tatsächlichen Verhältnissen zu machen». ${ }^{47}$

Mangelhafte Abstimmungen sind dann allenfalls aufzu- 38 heben, wenn die gerügten Mängel einen erheblichen Einfluss auf die Stimmberechtigten und das Ergebnis hatten. Es genügt grundsätzlich der Nachweis, dass eine derartige Beeinträchtigung möglich ist. ${ }^{48}$ Das Bundesgericht führt dazu aus, dass in quantitativer und qualitativer Hinsicht zu beurteilen sei, inwiefern die Schwere des festgestellten Mangels das Abstimmungsresultat beeinflusst hätte. Relevant sind hier die Rolle des Mangels im Abstimmungskampf und die Grösse des Stimmunterschieds. Es dürfen keine Zweifel an der Legitimität des Abstimmungsresultates entstanden sein und das Vertrauen in den demokratischen Prozess darf nicht verletzt sein. ${ }^{49}$ Kommt das Bundesgericht zum Schluss, dass eine Abstimmung ohne den Mangel anders ausgefallen wäre, kann die Abstimmung aufgehoben werden. 50

In der abwägenden Gesamtbetrachtung beim Entscheid 39 über die Aufhebung der Volksabstimmung über die Heiratsstrafe spielte insbesondere eine Rolle, dass die Abstimmung mit 50.8\% Nein-Stimmen äusserst knapp ausgefallen war, und dass eine Mehrheit der Kantone die Vorlage angenommen hatte. ${ }^{51}$ Zudem erklärte das Bundesgericht die allgemeine Informationslage im Vorfeld einer Volksabstimmung als justiziablen Gegenstand eines Verfahrens, womit indirekt auch die ansonsten vom zulässigen Anfechtungsobjekt ausgeschlossenen Abstimmungserläuterungen des Bundesrats erfasst sind. ${ }^{52}$

\section{Abstimmungserläuterungen im - nachträglichen - Faktencheck}

In den Abstimmungserläuterungen zum Referendum 40 über die EFTA-Indonesien Wirtschaftspartnerschaft wird

\begin{tabular}{l}
\hline 46 RHINOW et al. (Fn. 40), para. 2084; BGE113 Ia 291; BSK BV-TSCHAN- \\
NEN, Art. 34, N34. \\
\hline 47 BGE119 Ia 271 E. 3c. \\
\hline 48 BGE145 I 282 E.4.2. \\
\hline 49 BGE119 Ia 271 E.3b. \\
\hline 50 BGE145 I 207 E.4.1. \\
\hline 51 BGE145 I 207 E.4.3. \\
\hline 52 KASTRIOT LUBISHTANI / MAXIME FLATTET, La démocratie directe \\
face à la manipulation de l'information par des parcticuliers, AJP \\
2019, S.722; BIAGGINI (Fn. 2), S. 535.
\end{tabular}


konkret Bezug genommen auf den CEPA-Präferenzmechanismus für nachhaltig produziertes Palmöl. Während die Angaben dazu per se korrekt sind, wird darauf verzichtet, diese Angaben in einen Kontext zu stellen und beispielsweise zu erläutern, dass die gewährten Präferenzen nur max. 10\% der zu erwartenden Importe aus Indonesien erfassen. Folgende Angaben sind in den Abstimmungserläuterungen gemacht worden:

- Für den Import von indonesischem Palmöl sieht das Abkommen für eine beschränkte Menge gewisse Zollreduktionen vor. Wer Palmöl zu diesen Bedingungen importieren will, muss nachweisen, dass es unter Einhaltung der vereinbarten Umwelt- und Sozialauflagen produziert wurde. ${ }^{53}$

- Das Abkommen mit Indonesien enthält [...] spezifische Anforderungen an eine nachhaltige Palmölproduktion. So verpflichtet sich Indonesien, die Vorschriften zum Schutz der Urwälder und anderer Ökosysteme wirksam umzusetzen. ${ }^{54}$

- Die Zölle werden nicht aufgehoben, sondern nur gesenkt, und dies um rund 20 bis 40 Prozent. Diese Zollrabatte werden pro Jahr für höchstens 12'500 Tonnen gewährt. [...] Ein Importeur darf indonesisches Palmöl nur zu den tieferen Zöllen einführen, wenn es gemäss den vereinbarten Anforderungen an die Nachhaltigkeit produziert worden ist. ${ }^{55}$

41 Es ist zutreffend, dass die CEPA-Präferenzen nur gegen den Nachweis der Nachhaltigkeit gewährt werden. Verschwiegen wird allerdings, dass voraussichtlich rund 90\% der Importe aus Indonesien gar nicht vom CEPAPräferenzmechanismus erfasst werden (Futtermittel). Verschwiegen wird zudem, dass die $20-40 \%$ Zollrabatt auf Zolllinien Anwendung finden, welche bisher kaum eine Rolle gespielt haben bei der Einfuhr von Palmöl aus Indonesien. Zudem fehlt der Hinweis, dass wegen den betreffenden Zollbegünstigungscodes der Meistbegünstigtentarif im Hinblick auf die allermeisten Verwendungszwecke gar keine Anwendung findet und entsprechend gar nicht massgeblich ist.

Schliesslich fehlt auch der Hinweis, dass die maximal 12'500 Tonnen bis über 50\% der jährlichen Gesamteinfuhren an Palmöl ausmachen und einem x-Fachen der bisherigen Einfuhren an Palmöl aus Indonesien entsprächen. Hinzu kommt die Tatsache, dass nicht alle Einfuhren von Palmöl aus Indonesien von dieser Quote erfasst sind: Wie vorgängig erläutert, sind beispielsweise Einfuhren für Futtermittel nicht von den Mengenbeschränkungen in der EFTA-Indonesien Wirtschaftspartnerschaft

\footnotetext{
53 Bundeskanzlei, Volksabstimmung 7. März 2021, Erläuterungen des Bundesrates, 25. November 2020 (Abstimmungsunterlagen), S. 8. 54 Bundeskanzlei (Fn. 53), S. 46.

55 Bundeskanzlei (Fn. 53), S. 47.
}

erfasst. Der Verweis auf «beschränkte Mengen» erscheint demnach fragwürdig.

\section{Materielle Prüfung der Abstimmungsfreiheit im Referendum über die EFTA-Indonesien Wirtschaftspartnerschaft}

Der umfassende und unbehinderte Meinungsbildungs- 43 prozess setzt, wie oben erwähnt, voraus, dass die behördliche Informationstätigkeit den Grundsätzen der Transparenz, Verhältnismässigkeit und Sachlichkeit genügt. Die behördliche Informationstätigkeit im Abstimmungskampf des Referendums über die EFTA-Indonesien Wirtschaftspartnerschaft scheint grundsätzlich vereinbar mit dem Grundsatz der Transparenz: Es bestehen keinerlei Hinweise, dass die Behörden versucht hätten, diebehördliche Herkunft von Informationen zu verschleiern.

Zu prüfen ist sodann die Frage, ob ein Verstoss gegen die 44 Verhältnismässigkeit vorliegt, bzw. ob die behördliche Informationstätigkeit den Abstimmungskampf dominierte: Basierend auf den zur Verfügung stehenden Informationen war es den Stimmberechtigten zum Zeitpunkt der Abstimmung unmöglich, den CEPA-Präferenzmechanismus im Detail zu verstehen. Sowohl Pro- wie auch ContraArgumente bezogen sich ausschliesslich auf die vom Bundesrat zur Verfügung gestellten Informationen in Bezug auf die 20-40\% Zollrabatt und die «beschränkte Menge» von 12'500 Tonnen pro Jahr. Während die Details aufgrund der allgemein zur Verfügung stehenden Informationen hätten erarbeitet werden können, verlangt eine solche Kontextualisierung - wie die vorliegende rechtswissenschaftliche Fallstudie zeigt - Detailverständnis, welches nicht ohne Weiteres vorausgesetzt werden kann. Ein Rechtsgutachten, welches sich explizit mit dem Präferenzmechanismus in der EFTA-Indonesien Wirtschaftspartnerschaft befasste, klammerte die detaillierte Analyse des tatsächlichen ökonomischen Anreizes jedenfalls aus. ${ }^{56}$ Somit entsteht durchaus der Eindruck, dass über den Informationsvorsprung in dieser sehr spezifischen und komplexen Frage letztlich die behördliche Informationstätigkeit den Abstimmungskampf dominiert hat.

Bezüglich der Pflicht zur Sachlichkeit stellt sich die Fra- 45 ge, ob die Behörden im Abstimmungskampf über die EFTA-Indonesien Wirtschaftspartnerschaft über Zweck und Tragweite der Vorlage falsch orientiert haben oder wesentliche, für die Meinungsbildung bedeutsame Umstände nicht thematisiert haben. ${ }^{57}$ Die Erläuterungen in

56 Siehe BonANOMi (Fn. 29), S.10.

57 Laut Bundesgericht ist Sachlichkeit auch dann noch gegeben, wenn Informationen trotz einer gewissen Überspitzung nicht unwahr und unsachlich bzw. lediglich ungenau und unvollständig sind (BGE130 I 290 E.3.2); GIOVANNI BIAGGINI, BV Kommentar, 2. Aufl., Zürich 2017, Art. 34, S. 411. 
den Abstimmungsunterlagen sind korrekt und es bestehen auch keine Hinweise, dass die Behörden über den Zweck und die Tragweite der Vorlage insgesamt falsch orientiert hätten. Fraglich - auch in Anbetracht der Ergebnisse aus der Nachbefragung der Stimmberechtigten - bleibt, ob über den Verzicht der Kontextualisierung der «beschränkten Mengen», der 20-40\% Zollrabatte und des tatsächlichen Anwendungsbereichs des CEPA-Präferenzmechanismus für die Meinungsbildung bedeutsame Umstände nicht thematisiert worden sind. Die Rechtsprechung hat sich bisher noch nicht abschliessend $\mathrm{zu}$ einer vergleichbaren Rechtsfrage geäussert. ${ }^{58}$ Jedenfalls liegen keine offensichtlichen Gründe vor, die gegen eine differenziertere Darstellung des Zwecks und der Tragweite des CEPA-Präferenzmechanismus sprechen: Wie im ersten Teil dieses Artikels ausführlich beschrieben, vermag die Tatsache, dass der CEPA-Präferenzmechanismus den Charakter eines «nudges» trägt, zu überzeugen. Es leuchtet deshalb nicht ein, dass der Bundesrat darauf verzichtet hat, die regulatorische Errungenschaft des CEPAPräferenzmechanismus detailgetreu auszuführen. Es liegt gerade in der Natur der direkt-demokratischen Mitsprache, dass der Stimmbevölkerung auch komplexe Sachverhalte zugemutet werden. Eine Bevormundung der Stimmbevölkerung über allzu sehr vereinfachende Abstimmungserläuterungen fördert das Misstrauen in die behördliche Informationstätigkeit und gefährdet somit die faktenbasierte Grundlage des politischen Meinungsbildungsprozesses. Die Sorgfaltspflicht in Bezug auf Sachlichkeit behördlicher Informationstätigkeit muss daher hoch angesetzt werden.

Entsprechend liegen Mängel vor in Bezug auf die Verhältnismässigkeit und die Sachlichkeit der behördlichen Informationstätigkeit im Rahmen des Referendums über die EFTA-Indonesien Wirtschaftspartnerschaft, insbesondere in Bezug auf den CEPA-Präferenzmechanismus. Bezüglich dieses Teilaspekts der Vorlage erscheint demnach eine Verletzung von Art. 34 Abs. 2 BV gegeben.

\section{Stimmrechtsbeschwerde gegen das Referendum über die EFTA-Indonesien Wirtschaftspartnerschaft?}

47 Die EFTA-Indonesien Wirtschaftspartnerschaft wurde am 7. März 2021 mit 51.65\% der Stimmen und einer klaren Mehrheit der Kantone angenommen. Der Stimmrechtsunterschied beträgt knapp 90’oooStimmen. ${ }^{59} \mathrm{Ge}-$ mäss der VOX-Analyse zum Referendum vom 7. März 2021 waren für 12\% der Ja-Stimmenden Überlegungen zur

\footnotetext{
58 Siehe auch Bundeskanzlei (Fn. 53), S. 413.

59 Bundeskanzlei, Vorlage Nr.640, Provisorisches amtliches Ergebnis, Bundesbeschluss über die Genehmigung des Umfassenden Wirt schaftspartnerschaftsabkommens zwischen den EFTA-Staaten und Indonesien.
}

Umwelt (Umweltstandards im Abkommen; nachweislich nachhaltiges Palmöl importieren; ohne Abkommen wird der Umwelt noch mehr geschadet) ausschlaggebend. ${ }^{60}$ Umweltanliegen haben zudem am stärksten polarisiert. Dem Argument, das Freihandelsabkommen sei fortschrittlich, da es zur nachhaltigen Entwicklung der Umwelt und von Indonesien beiträgt, stimmten 80\% der Ja-Stimmenden zu, während 74\% der Nein-Stimmenden das Argument verwarfen. ${ }^{61}$ Die oben festgestellten Mängel in Bezug auf die allgemeine Informationslage über den CEPA-Präferenzmechanismus für nachhaltig produziertes Palmöl haben somit potentiell das Abstimmungsergebnis tangiert.

Die hier aufgeführten Erläuterungen zu den Details des 48 CEPA-Präferenzmechanismus waren zur Zeit der Abstimmung in dieser Form nicht bekannt. Sie stellen somit grundsätzlich Fakten dar, die bereits vorhanden, aber noch unbekannt waren (sog. unechte Noven). ${ }^{62}$ Im Rahmen eines nachträglich, wiedererwägungsweise geltend gemachten Rechtsschutzes basierend auf einer Verletzung von Art. 34 Abs. 2 BV müsste allerdings nachgewiesen werden, dass die Informationen über die Details des CEPA-Präferenzmechanismus nicht ohne Weiteres in die öffentliche Diskussion hätten eingebracht werden können, und dass die allgemeine Informationslage ungenügend war. 63

Die Frage danach, wieviel indonesisches Palmöl proJahr, 49 für welchen Verwendungszweck und mit welchem konkreten Präferenzzoll in Zukunft in die Schweiz würde importiert werden können, liegt auf der Hand, wurde aber scheinbar im Abstimmungskampf nicht aufgeworfen. Ob dies daran liegt, dass der Thematik des CEPA-Präferenzmechanismus im Abstimmungskampf sowieso mehr symbolischer Charakter zukam - in der Form einer Stellvertreter-Diskussion an Stelle der grundlegenderen Frage nach Sinn und Zweck von Freihandelsabkommen -, oder daran, dass die behördlichen Informationen fälschlicherweise als vollständig erachtet wurden, ist unklar. Die Vorlage befasste sich allerdings mit der Ratifikation der EFTA-Indonesien Wirtschaftspartnerschaft als Gesamtes. Allein aufgrund mangelnder Detailkenntnisse des CEPA-Präferenzmechanismus kann die Informationslage folglich nicht unbedingt als ungenügend erachtet werden. Hier unterscheidet sich der vorliegende Fall von der Ausgangslage in der Rechtsprechung betreffend die Volksabstimmungen über die Unternehmenssteuerreform ${ }^{64}$

60 VOX-Analyse März 2021, Nachbefragung und Analyse zur eidgenössischen Volksabstimmung vom 7. März 2021, gfs.bern, April 2021, S. 37. 61 VOX-Analyse (Fn. 60), S. 39.

62 Vergleiche dazu BGE138I61 E. 4.5.

63 Siehe auch Urteil des Bundesgerichts 1C_713/2020,1C_715/2020 vom 23. März 2021 E. 4.1.3f.

64 BGE138I61. 
und gegen die Heiratsstrafe ${ }^{65}$ : Die mangelhafte behördliche Informationstätigkeit bezieht sich hier auf einen bestimmten Teilaspekt der Vorlage, während es sich dort um Informationen handelte, welche den Zweck und die Tragweite der Vorlage wesentlich veränderten.

Es ist folglich möglich bis wahrscheinlich, dass die prozessuale Durchsetzung der Verletzung von Art. 34 Abs. 2 BV daran scheitert, dass in Bezug auf Zweck und Tragweite der Abstimmungsvorlage die allgemeine Informationslage nicht als ungenügend erachtet werden kann. Daran vermag auch die Tatsache, dass Nachhaltigkeitsüberlegungen betreffend den Import von Palmöl wesentlich waren für geschätzte $12 \%$ der Ja-Stimmenden, nichts zuändern: Es war zum Zeitpunkt der Abstimmung allgemein bekannt, dass es ungewiss bleibt, ob der Präferenzmechanismus tatsächlich zu einer nachhaltigeren Palmölproduktion in Indonesien führt. ${ }^{66}$ Zweck und Tragweite des Nachhaltigkeitskapitels in der EFTA-Indonesien Wirtschaftspartnerschaft waren somit grundsätzlich bekannt.

\section{Vermeidung künftiger Verletzungen der Abstimmungsfreiheit}

51 Nichtsdestotrotz gilt es insbesondere im Hinblick auf künftige Volksabstimmungen über Freihandelsabkommen, die behördliche Informationspraxis zu verbessern, um Verletzungen der Abstimmungsfreiheit zu vermeiden. Relevant ist in dieser Hinsicht, dass die Rechtsprechung unterscheidet zwischen der Vorbereitungsphase auf eine Abstimmung und dem eigentlichen Abstimmungskampf. Das Bundesgericht geht grundsätzlich davon aus, dass bereits in der Vorbereitungsphase alle wesentlichen, für die Meinungsbildung bedeutsamen Informationen zur Verfügung gestellt worden sind. Den Behörden ist daher während dem Abstimmungskampf Zurückhaltung in der Informationstätigkeit geboten, weil eine solche auch leicht in einer unzulässigen «Einmischung» in den Meinungsbildungsprozess resultieren kann. ${ }^{67}$ Umso wichtiger wäre es daher gewesen, dass die spezifischen Details des CEPA-Präferenzmechanismus bereits in den vorbereitenden Beratungen in den zuständigen Kommissionen der Bundesversammlung bekannt gemacht worden wären. Ein allzu grosser Informationsvorsprung der Bundesverwaltung gegenüber der Legislative lässt sich nicht innerhalb eines Abstimmungskampfes überwinden und stört somit unvermeidlich den Meinungsbildungsprozess.

65 BGE145I207.

66 Siehe auch BonANOMI (Fn. 29).

67 BGE121I252, Regeste 2. Leitsatz.
Insbesondere weil die Volksabstimmung über die EFTA- 52 Indonesien Wirtschaftspartnerschaft auch die erste derartige Abstimmung war seit der Volksabstimmung über das Freihandelsabkommen mit der heutigen EU $1972^{68}$, konnte nicht auf ein allgemeines Vorwissen der Stimmberechtigten über den Aufbau und Inhalt von internationalen Handelsabkommen aufgebaut werden. Umso wichtiger erscheint es daher für die Gewährleistung der Abstimmungsfreiheit, dass angesichts der Abkehr von der sog. Standardabkommen-Praxis ${ }^{69}$ umfassender, pro-aktiver und vorzeitiger über internationale Handelsabkommen informiert wird.

So verbleiben beispielsweise die öffentlich zugänglichen 53 Informationen über laufende und abgeschlossene Verhandlungen über Freihandelsabkommen der Schweiz dürftig. Auf Nachfrage und im Rahmen institutionalisierter Treffen sind die wesentlichen Informationen sehr wohl zugänglich, jedoch werden diese selten proaktiv geteilt. Es überrascht, dass beispielsweise die EUKommission $^{70}$, Neuseeland ${ }^{71}$ und Kanada ${ }^{72}$ pro-aktiver und umfassender informieren über geplante und laufende Verhandlungen als die Schweiz. ${ }^{73}$ Was im Vergleich mit den Genannten in der Schweiz fehlt, sind insbesondere die Offenlegung des Verhandlungsmandats (wenigstens in groben Zügen), der Entwürfe für Abkommenstexte, der exploratorischen Gespräche und der Inhalte und Fortschritte laufender Verhandlungen. So wird gegenwärtig mit Malaysia unter anderem über den präferenziellen Marktzugang von nachhaltig produziertem Palmöl verhandelt. Während auf der schweizerischen Seite nicht über den Fortschritt der Verhandlungen berichtet wird, findet sich auf der Website der EFTA eine Zusammenfassung der 10. Verhandlungsrunde vom Mai 2021, worin allerdings der Stand der Verhandlungen in Bezug auf nachhaltig produziertes Palmöl unerwähnt bleibt. ${ }^{74}$ Ein Umdenken im Hinblick auf pro-aktive, frühzeitige und umfassende behördliche Information über Freihandelsabkommen drängt sich nur allein schon deswegen auf, weil seit der Abkehr von der sog. Standardabkommen-Praxis die Wahrscheinlichkeit gross ist, dass in einer Volksabstimmung über das Verhandlungsresultat entschieden werden wird.

68 Abkommen zwischen der Schweizerischen Eidgenossenschaft und der Europäischen Wirtschaftsgemeinschaft, abgeschlossen am 22.Juli 1972 (SR 0.632.401).

69 Siehe z.B. GEBISTORF (Fn. 37), S. 463.

70 Siehe Portal der EU-Kommission zum internationalen Handel.

71 Siehe Portal des neuseeländischen Ministeriums für Foreign Affairs \& Trade zur neuseeländischen Handelspolitik.

72 Siehe Portal der kanadischen Regierung zu Trade and Investment Agreements.

73 Siehe Portal zur Aussenwirtschaft \& Wirtschaftlichen Zusammenarbeit des Staatssekretariats für Wirtschaft SECO.

74 EFTA, Negotiations between the EFTA States and Malaysia on an Economic Partnership Agreement, 10th Round of Negotiations, 25. 27. und 28. Mai 2021. 
54 Aber auch nach der eigentlichen Vorbereitungsphase besteht Verbesserungsbedarf. So wäre gegebenenfalls zu prüfen, bis zu welchem Grad vereinfachende Information überhaupt mit den Grundsätzen der Sachlichkeit, Transparenz und Verhältnismässigkeit in der behördlichen Informationstätigkeit vereinbar ist. Wichtig erscheint insbesondere, dass den Stimmberechtigten die Komplexität einer Abstimmungsvorlage nicht vorenthalten wird, auch wenn dies allenfalls zu umfangreicheren Abstimmungserläuterungen führt, bzw. einen Mehraufwand an zusätzlicher Bereitstellung von Fakten nach sich zieht.

\section{Schlussfolgerungen}

Der Abstimmungskampf über die EFTA-Indonesien Wirtschaftspartnerschaft illustriert eindrücklich, wie substantiell sich der Informationsvorsprung zwischen der Bundesverwaltung und der Legislative unter Umständen auf den politischen Meinungsbildungsprozess auswirken kann. In einer zentralen Streitfrage kamen relevante Fakten nicht zur Sprache. Unter anderem lässt sich dies damit erklären, dass die sog. Standardabkommen-Praxis erst kürzlich aufgegeben wurde und damit der politische Meinungsbildungsprozess in der Schweiz noch nicht à jour ist in Bezug auf die mit internationalen Handelsabkommen einhergehende regulatorische Komplexität.

Anderseits mag dies auch daran liegen, dass in diesem ganz speziellen Fall die involvierten politischen Akteure gar kein tatsächliches Interesse daran hatten, die Details des präferenziellen Marktzugangs für nachhaltig produziertes indonesisches Palmöl offenzulegen. Es wäre daher falsch, die politische Verantwortung für die Gewährleis- tung der Abstimmungsfreiheit alleine dem Bundesrat zuzuschieben. Trotz der festgestellten Verletzung von Art. 34 Abs. 2 BV liegt eher keine ungenügende Informationslage in Bezug auf Zweck und Tragweite des Referendums über die EFTA-Indonesien Wirtschaftspartnerschaft als Gesamtes vor.

Gerade weil sich die ökonomischen Anreize des CEPA- 57 Präferenzmechanismus bei näherem Hinsehen als geringfügig herausstellen, liegt es durchaus im Rahmen des Möglichen, dass er den politischen Druck auf andere WTO-Mitgliedstaaten erhöht, ähnliche Präferenzmechanismen in ihre Handelspartnerschaften aufzunehmen. Während politisch ein Zeichen gesetzt wird, wird dieses ökonomisch nur über einen «nudge» durchgesetzt. Der CEPA-Präferenzmechanismus leistet damit einen wesentlichen Beitrag für einen künftigen neuen globalen Konsens über zentrale Mindestauflagen für nicht-produktbezogene Verfahren und Produktionsmethoden. Weshalb dies in den Abstimmungserläuterungen nicht so kommuniziert worden ist, bleibt unersichtlich.

Um die Abstimmungsfreiheit in der politischen Entscheid- 58 findung über internationale Handelsabkommen besser gewährleisten zu können, muss die behördliche Informationstätigkeit in der Vorbereitungsphase gestärkt werden und pro-aktiver, umfassender und vorzeitiger informiert werden. Nur so kann vermieden werden, dass aufgrund von Druck zur Vereinfachung und Knappheit behördlicher Informationstätigkeit für die Meinungsbildung bedeutsame Umstände nicht thematisiert werden und gleichzeitig diese knappen Behördeninformationen den Diskurs im Abstimmungskampf mangels Alternativen dominieren. 